\title{
Application of Overlapping Mesh in Numerical Hydrodynamics
}

\author{
Roozbeh Panahi, Assoc. Prof. \\ Mehdi Shafieefar, Assoc. Prof. \\ Tarbiat Modares University
}

\begin{abstract}
A Finite Volume $(F V)$ algorithm is presented to investigate two-dimensional hydrodynamic problems including viscous free surface flow interaction with free rigid bodies in the case of large and/or relative motions. Two-phase flow with complex deformations at the interface is simulated using a fractional stepvolume of fluid algorithm while it is also capable of representing a high quality wave tank, according to implemented temporal discretisation. Rigid body motions are also captured using two overset meshes. Flow variables are transferred using a simple fully implicit non-conservative interpolation scheme which maintains the second-order accuracy of implemented spatial discretisation. A code is developed and an appropriate set of problems are investigated. Results show a good potential to develop a virtual hydrodynamics laboratory.
\end{abstract}

Keywords: finite volume; two-phase flow; overlapping mesh; fluid-structure interaction

\section{INTRODUCTION}

There is an increasing demand to facilitate the use of marine environment by planning onshore infrastructures, offshore platforms, high speed vessels, etc., which are all along with high costs in design as well as in construction. Computational Fluid Dynamics (CFD) shortens the way to meet many design requirements in hydrodynamics. Here, a unified algorithm is developed and examined to tackle hydrodynamic problems including fluid-structure interaction with characteristics indicated in Tab. 1.

However, one encounters three major difficulties to make decision about them in a numerical algorithm to solve such problems. They are presented in the first row of Tab. 2 . Available approaches and selected methods based on covered hydrodynamics problems specifications (Tab. 1) are also summarized in second and third row of this Table.

Governing equations are reviewed in section 2. They have to be revised according to requirements in simulation of two-phase flow using FV discretisation in moving meshes. Section 3 is dedicated to discretisation. Solution of the Navier-Stokes equations is discussed in section 4. Section 5 is devoted to overlapping mesh motion modeling strategy. A general algorithm to concisely show the relation between different parts of developed fluid-structure interaction solver is presented in section 6 . The present procedure is coded and verified in section 7 by applying it to some flows for which either numerical solution or experimental data are available.
Tab. 1. Scope of encountered problems including fluid-structure interaction in the context of an interfacial flow

\begin{tabular}{|c|c|}
\hline Fluid and Flow & Structure and Motion \\
\hline without surface tension & 2D \\
\hline $\begin{array}{c}\text { with homogenous property } \\
\text { distribution }\end{array}$ & fixed/free(3-DoF) \\
\hline without suspended particles & floating/submerged \\
\hline Incompressible & large amplitude motions \\
\hline Viscous & Rigid \\
\hline Rotational & with relative motions \\
\hline Newtonian & $\begin{array}{c}\text { with auxiliary equipments } \\
\text { propeller, rudder, mooring }\end{array}$ \\
\hline steady/unsteady
\end{tabular}

Potentials of the algorithm are demonstrated where complex interfacial flow, relative and/or large amplitude motions as well as wave generation and propagation are of interest. 
Tab. 2. Encountered problems, available approaches and implemented methods

\begin{tabular}{|c|c|c|}
\hline Case & Available approaches & Implemented approach \\
\hline $\begin{array}{c}\text { Coupling of the pressure and the } \\
\text { velocity fields } \\
\text { (Ferziger and peric, 2002) }\end{array}$ & $\begin{array}{c}\text { Predictor-corrector } \\
\text { Artificial compressibility } \\
\text { Fractional step }\end{array}$ & Fractional step \\
\hline $\begin{array}{l}\text { Simulation of free surface } \\
\text { (Ubbink and issa, 1999) }\end{array}$ & $\begin{array}{l}\text { Surface tracking } \\
\text { Surface capturing }\end{array}$ & Surface capturing \\
\hline $\begin{array}{l}\text { Capturing of rigid body motions in } \\
\text { a domain }\end{array}$ & $\begin{array}{c}\text { Body-attached/moving mesh (panahi et al., 2006A) } \\
\text { Deformable mesh (chentanez et al., 2006) } \\
\text { Re-mesh (tremel et al., 2007) } \\
\text { Sliding mesh (blades and marcum, 2007) } \\
\text { Overlapping mesh (carrica et al., 2007) } \\
\text { Cartesian mesh (mittal and iccarino, 2005) }\end{array}$ & Overlapping mesh \\
\hline
\end{tabular}

\section{GOVERNING EQUATIONS}

According to characteristics of the encountered two-phase flow field, see Tab. 1, and implemented motion modeling strategy, the following set of equations in the Arbitrary Lagrangian-Eulerian (ALE) Cartesian form are used:

$$
\begin{gathered}
\frac{d}{d t} \int_{V} \rho_{\text {eff }} d V+\int_{A} \rho_{\text {eff }} \mathbf{u}_{\text {rel }} \cdot \mathbf{n} \mathrm{dA}=0 \\
\frac{\mathrm{d}}{\mathrm{dt}} \int_{\mathrm{V}} \mathrm{u}_{\mathrm{i}} \mathrm{dV}+\int_{\mathrm{A}} \mathrm{u}_{\mathrm{i}}\left(\mathbf{u}_{\text {rel }} \cdot \mathbf{n}\right) \mathrm{dA}= \\
=-\int_{\mathrm{A}} \frac{1}{\rho_{\text {eff }}} \mathrm{Pn}_{\mathrm{i}} \mathrm{dA}+\int_{\mathrm{A}} v_{\mathrm{eff}} \vec{\nabla} \mathrm{u}_{\mathrm{i}} \cdot \mathbf{n} \mathrm{dA}+\int_{\mathrm{V}} \mathrm{g}_{\mathrm{i}} \mathrm{dV} \\
\frac{\mathrm{d}}{\mathrm{dt}} \int_{\mathrm{V}} \alpha \mathrm{dV}+\int_{\mathrm{A}} \alpha \mathbf{u}_{\text {rel }} \cdot \mathbf{n} \mathrm{dA}=0
\end{gathered}
$$

where:

$\rho_{\text {eff }}=\alpha \rho_{1}+(1-\alpha) \rho_{2}$ and $v_{\text {eff }}=\alpha v_{1}+(1-\alpha) v_{2}$ are density and dynamic viscosity of an effective phase as a combination of phases volume fraction $\alpha$. It is obvious that $\alpha$ is calculated in each Control Volume (CV) by solving a transport equation. Volume fraction of zero in a CV indicates the presence of one fluid and the unity indicates the other fluid; $\mathbf{u}_{\mathrm{rel}}=\mathbf{u}-\mathbf{u}_{\mathrm{m}}$ is the fluid velocity vector $\mathbf{u}$ relative to the mesh velocity vector $\mathbf{u}_{\mathrm{m}}$ and $\mathbf{n}$ represents a unit vector normal to a CV face. $u_{i}$ is the velocity component in the $\mathrm{i}$ Cartesian direction, $\mathrm{P}$ stands for the pressure, $n_{i}$ is the i Cartesian direction component of $\mathbf{n}$ and $\mathrm{g}_{\mathrm{i}}$ indicates the gravity component in this direction.

Movements of a free rigid body are also included in this study. They are calculated based on loads acting on the body, by solving the linear and angular momentum equations. Such loads can be raised from effects of flow field, body weight and probably external components. Rigid body motion equations are treated in a Global Coordinate System (GCS); a nonrotating, non-accelerating Newtonian reference system.

\section{DISCRETISATION}

Here, discretisation of all differential governing equations is reviewed. More details can be found in a recent paper of the authors (Jahanbakhsh et al., 2007).

\section{Momentum Conservation Equations}

On the 1.h.s of Eq.(2), the simplest approximation for the spatial discretisation of the first term (unsteady term) is to replace it by the product of the value of the integrand at the $\mathrm{CV}$ center and the volume of the CV. Convection term (the second term) is also discretised using Gamma interpolation (Jasak, 1996). Besides, on the r.h.s. of Eq.(2), using the common Linear Interpolations (LI) to discretise pressure term (the first term) results in oscillations in the velocity field in the case of twophase flow. Such oscillations lead the solution to a divergence, especially when there are two phases with a high density ratio e.g. water and air. Here, a Piecewise LI (PLI) is implemented, recently introduced by the authors (Jahanbakhsh et al., 2007). The second term (diffusion term) is treated by over-relaxed interpolation (Jasak, 1996). Finally, the last term (gravity term) is discretised as the unsteady term.

Temporal discretisation of the momentum conservation equations is in direct relation to the way that the pressure and the velocity fields are coupled. So, it is discussed together with implemented fractional step method in the next section.

\section{Volume Fraction Transport Equation}

Spatial discretisation of the unsteady term is done similar to that of the momentum conservation equations. About its temporal discretisation, although the first-order Euler implicit interpolation is the obvious choice but it has been shown in the numerical results that such a temporal discretisation is not a good option when wave generation and propagation are the case. In contrast, the second-order three-time-levels temporal discretisation proposes a minimum level of diffusion in such problems. The Compressive Interface Capturing Scheme for Arbitrary Meshes (CICSAM) (Ubbink and Issa, 1999) is used for spatial discretisation of the convection term as well as Crank-Nicholson interpolation for its temporal discretisation according to an investigation conducted by the authors (Panahi et al., 2005).

\section{COUPLING OF THE PRESSURE AND THE VELOCITY FIELDS}

To compute the pressure and the velocity fields, the fractional step method of Kim and Choi (Kim and Choi, 2000) is implemented. Here, Crank-Nicholson scheme is used for the temporal discretisation of the convection term in contrast to Adams-bashforth scheme used in the original method. In addition, convection term is linearized using Picard iteration method instead of the Newton's method in (Kim and Choi, 2000). Such an algorithm can be found as a flowchart in (Panahi et al., 2006a).

\section{OVERLAPPING MESH}

As mentioned earlier, to simulate fluid-structure interaction including moving bodies, a motion modeling strategy is 
necessary in addition to an interfacial flow solver. Here, an overlapping mesh system; a strategy among non-domainconforming motion modeling strategies; also known as overset or chimera mesh is implemented. Using such a strategy, the computational domain is covered by a number of boundaryfitted overlapping meshes (mesh components), in general. Mesh components associated with moving structures move with them, as in the case of a body-attached mesh motion modeling strategy, while the other mesh components remain stationary. An overlapping mesh system is shown in Fig. 1 consisting of two mesh components.
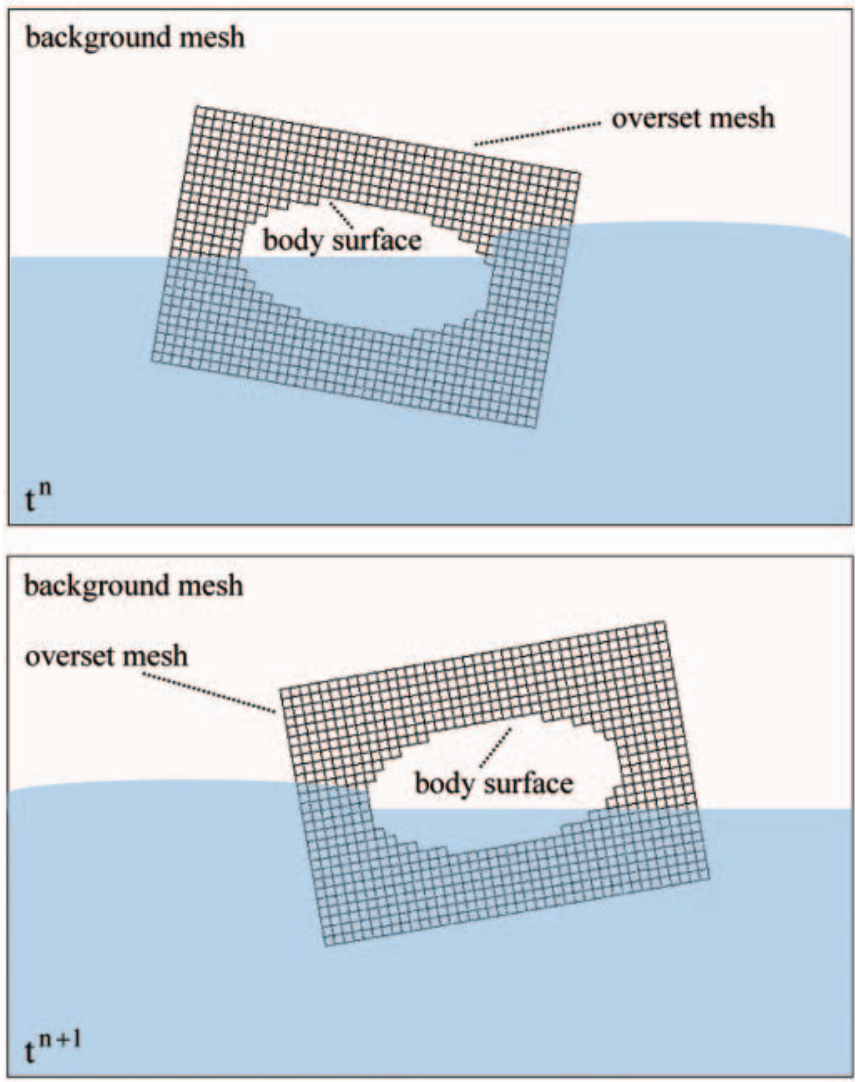

Fig. 1. Overlapping mesh motion modeling strategy including two mesh components; computational domain in two successive time steps $t^{n}$ and $t^{n+1}$ where the overset mesh follows the moving body while the background mesh remains stationary

The mesh components are not required to match in any especial way, but they have to overlap sufficiently to provide the means of coupling the solution on each of them. This method allows the mesh components to move relative to each other in an arbitrary fashion, making it prefect for use in applications with moving bodies. The mesh components are usually geometrically simple and allow for independent meshing of higher quality than would be possible in the case of a single mesh.

Here, flow variables have to be interpolated between the overlapped meshes to exchange the information. The major drawback of this approach is however the difficulty to ensure conservation of the computed variables, which can be neglected in many cases (Togashi et al., 2001; Hadzic, 2005) as presented in this study.

The overlapping mesh computation was performed firstly in 1981 to facilitate mesh generation in the case of complex boundaries (Atta, 1981). It was later used to predict forced relative motions (Buning et al., 2000) and also aerodynamic problems (Chen et al., 2000). It is just recently used in marine applications due to difficulties with an interfacial flow (Carrica et al., 2007).
The utilized overlapping mesh motion modeling strategy consists of three distinct steps which will be discussed in the following sub-sections.

\section{Identification of CVs}

When all mesh components necessary to appropriately cover the computational domain are generated, the next step is to identify the characteristic of all CVs according to their role in the solution process (Hadzic, 2005):

+ discretisation cells which are used to discretise the governing equations

+ interpolation cells which receive the solution from the overlap mesh component by interpolation

+ inactive cells which are disregarded during the solution process

The main activity toward marking all cells in this step is called "hole cutting". It is generally implemented for the background mesh. For computations in the case of moving bodies, it is important that the hole cutting can be performed both automatically and rapidly, because identities of CVs have to be updated in each time step. For complex configurations, this may become difficult and more general algorithms may be $\mathrm{n}$ ecessary to accomplish an automatic hole cutting (Nakahashi et al., 2000; Meakin, 2001; Suhs et al., 2002).

To explain the algorithm developed in this study, consider the overlapping mesh schematically shown in Fig. 2. It consists of two mesh components: a background mesh in the whole
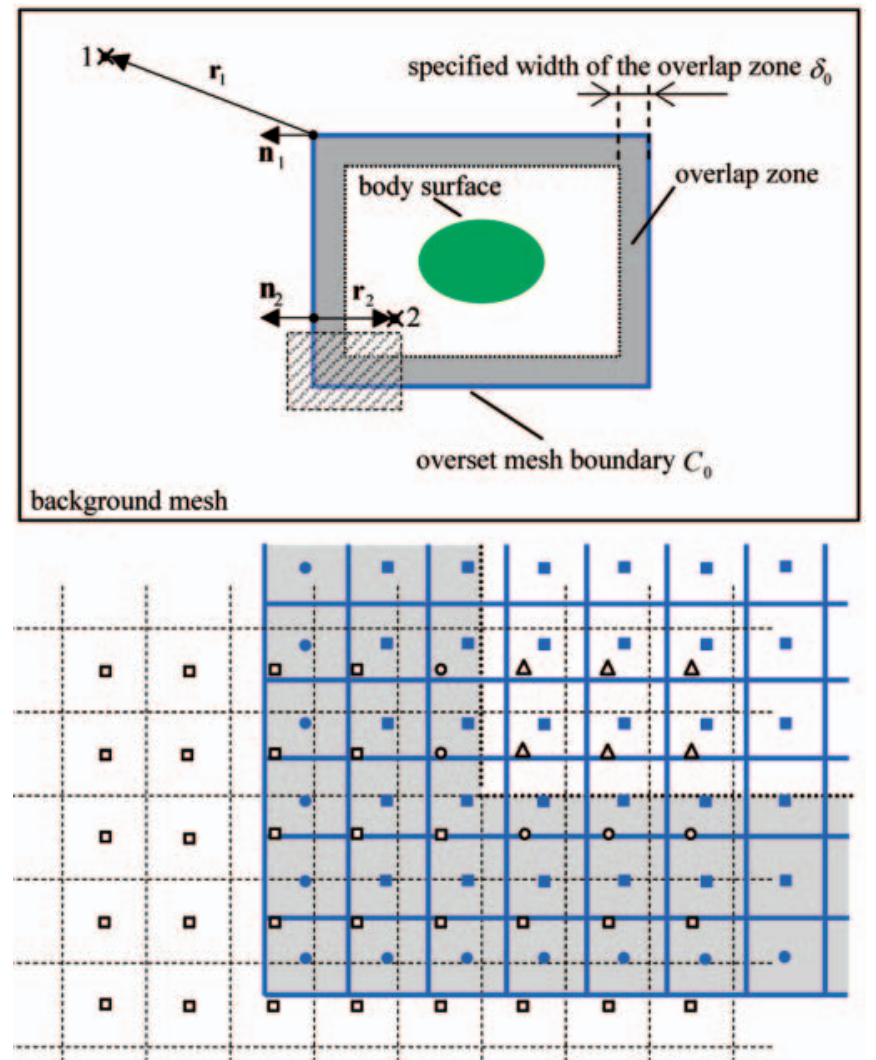

a discretization CV of the background mesh

- interpolation CV of the background mesh

$\triangle \quad$ inactive $\mathrm{CV}$ of the background mesh

- interpolation CV of the overset mesh

- discretization CV of the overset mesh

Fig. 2. Identification of $C V$ s; (up): hole cutting procedure for two typical cell centers 1 and 2 in the background mesh, respectively inside and outside of the overset mesh, (down): hatched area of the left figure when the identification procedure is completed 
computational domain and an overset mesh around the body. The procedure to identify all cells in the background mesh is noticeably shown in Fig. 3. It must be mentioned that, the width of the overlap zone $\left(\delta_{0}\right)$ depends on the mesh spacing in this area. It has to be large enough to provide sufficient overlap between the meshes as an essential element to have an appropriate inter-mesh coupling.

As aforementioned, hole cutting is just implemented to identify cells in the background mesh. About an overset mesh, type classification is very simple. During the first step and mesh generation process, outer boundary of the overset mesh is assigned a special boundary type (overset mesh boundary). Then, the CVs that lie along such a boundary are recognized as interpolation cells. Other CVs in the overset mesh are discretisation cells.

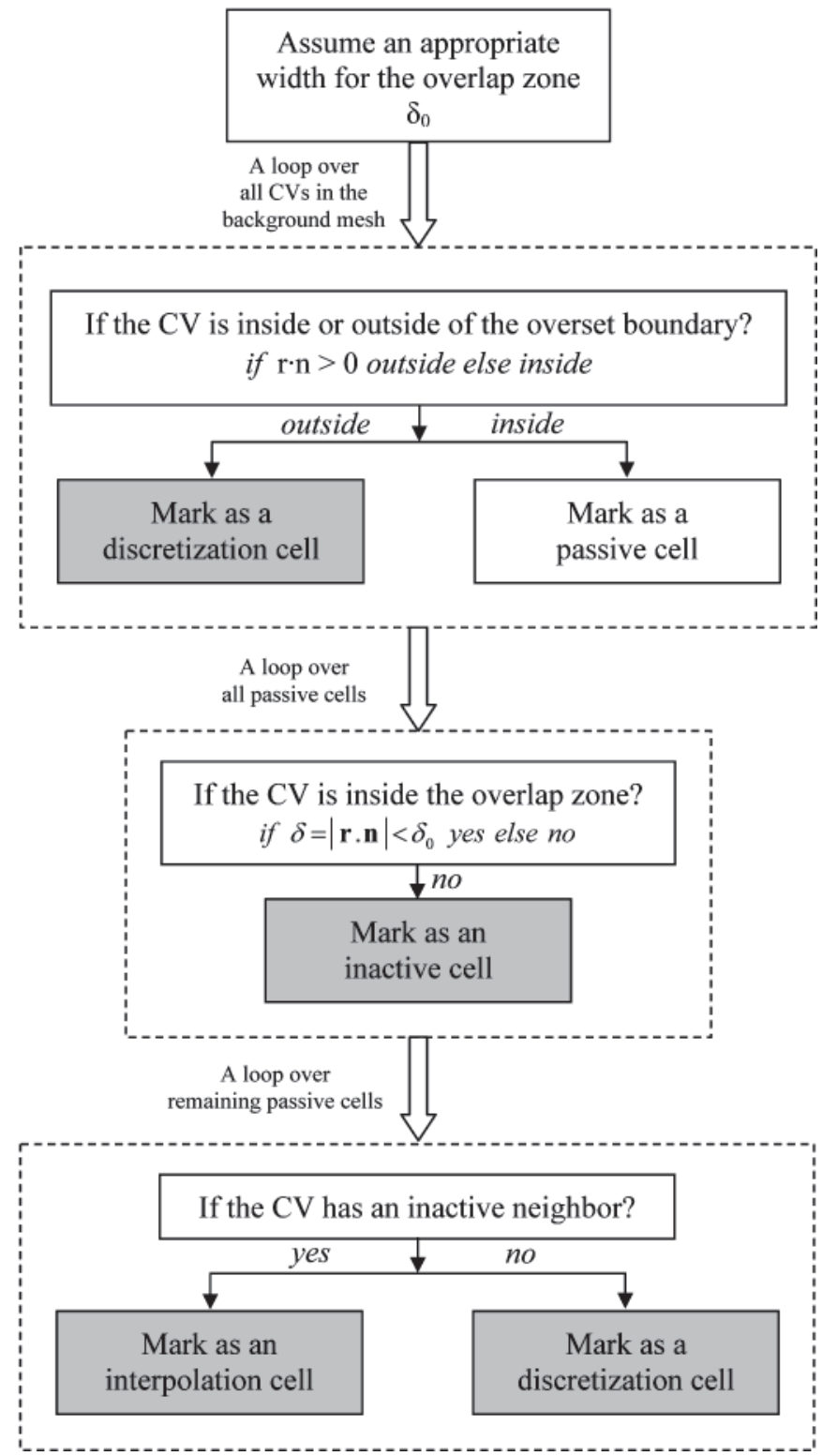

Fig. 3. An algorithm to identify CVs in the background mesh; grey squares present when a decision is made about the identity of a $\mathrm{CV}$

\section{Coupling of Mesh Components}

Here, a non-conservative interpolation approach is employed to make a unique solution by transferring all flow variables. In other words, a variable at an interpolation cell of a mesh component; identified in the previous step for both background and overset meshes; is obtained by interpolation of the variable from the overlapped mesh. The later mesh is called the donor mesh. Therefore, an interpolation stencil must be constructed for each interpolation cell of the considered mesh from $\mathrm{CVs}$ of the corresponding coincident mesh (donor mesh). A CV whose center is closest to the center of the interpolation cell (host cell) is the base of an interpolation stencil. Any additional cells on the donor mesh contributing to the interpolation formula come from the immediate neighborhood of the host cell. That is, the main operation in this step is called "host searching".

In the present study a neighbor-to-neighbor searching algorithm (Löhner, 1995), suitable for unstructured meshes, is employed to accomplish the task. The method is schematically shown in Fig. 4. Starting from a given CV (starting CV), one jumps to the neighboring $\mathrm{CV}$ that lies in the direction of the target cell center. This procedure is repeated until the $\mathrm{CV}$ which contains the target cell (interpolation cell) center is reached. Selection of the next starting CV among the neighbor CVs of the current starting one is based on $\mathbf{p}_{\mathrm{i}} \cdot \mathbf{n}_{\mathrm{j}} ; \mathbf{p}_{\mathrm{j}}$ is a vector connecting the midpoint of each face into the target cell center, $\mathbf{n}_{\mathrm{j}}$ is an outward normal vector on the face of the present starting $\mathrm{CV}$ and $\mathrm{j}$ is the face counter. The face whose normal encloses the smallest angle with vector $\mathbf{p}_{\mathrm{j}}$ is selected and the neighboring $\mathrm{CV}$ that shares this face with the present starting $\mathrm{CV}$ is chosen as the new starting $\mathrm{CV}$ in the donor mesh. If the dot products are negative for all faces of a $\mathrm{CV}$, the target cell center lies inside that $\mathrm{CV}$, i.e. the host cell is found.
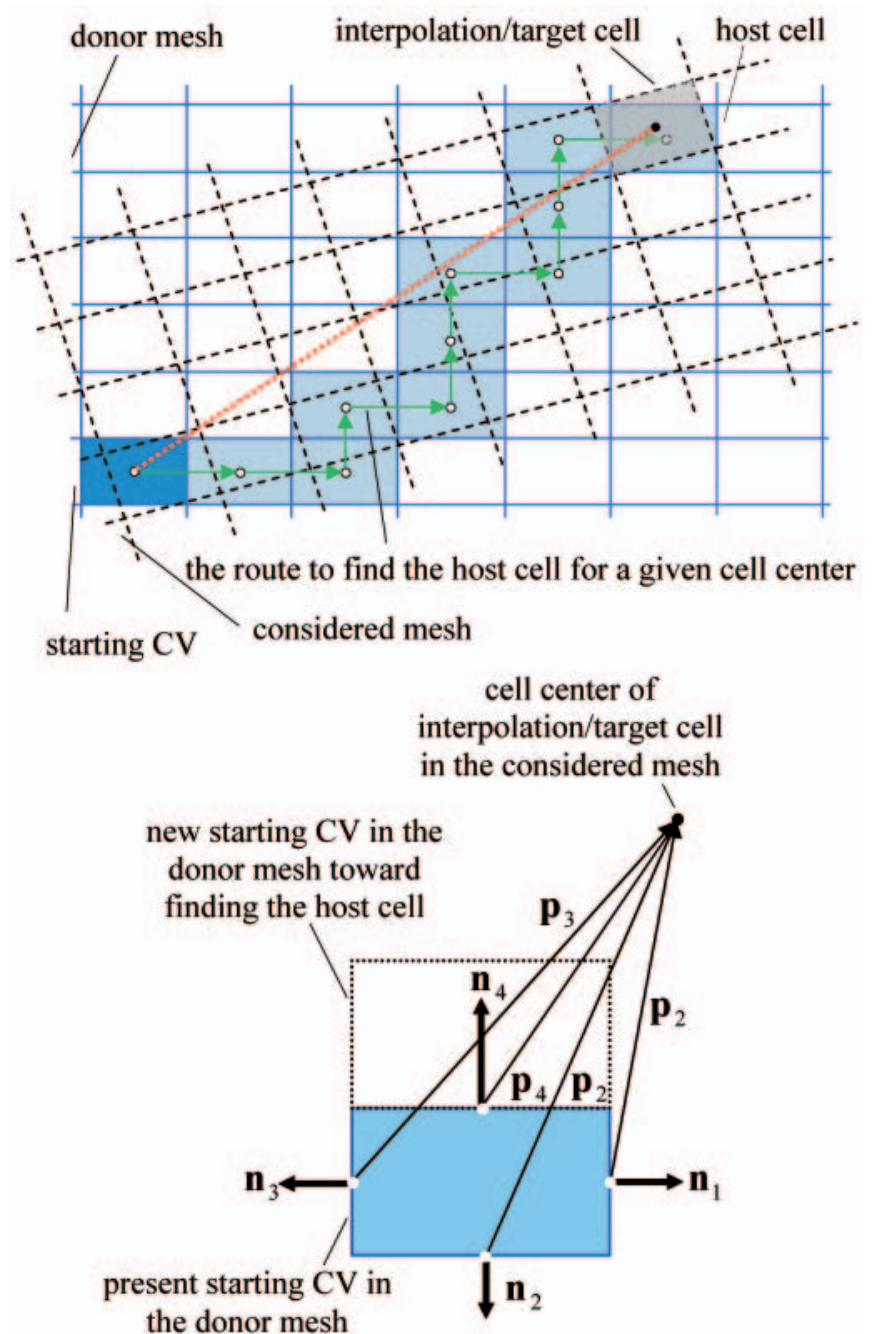

Fig. 4. Donor searching to find host cells in donor mesh for all interpolation cells of considered mesh; (up): a route to find the host cell for a given interpolation cell, (down): making a decision to continue the way toward finding a host cell in the donor mesh for a given cell center, based on $\mathbf{p}_{j} \cdot \mathbf{n}$ at face centers 
This searching algorithm is very efficient, since the searching path is one-dimensional even on a $3 \mathrm{D}$ mesh made of arbitrary polyhedral CVs. While body movements are small within a time step, the new host cell for each interpolation cell usually lies in the immediate neighborhood of the previous one. This reduces the searching time in each time step.

In this study, construction of an interpolation formula consists of four neighbor CVs in addition to the host cell for all flow variables, see Fig. 5. According to this figure, a fully implicit algebraic equation for an interpolation cell is created as below for variable $\varphi$ which is velocity components ( $u$ and $\mathrm{w})$, pressure (P) and also volume fraction $(\alpha)$ :

$$
\varphi_{\mathrm{I}}=\varphi_{\mathrm{H}}+(\vec{\nabla} \varphi)_{\mathrm{H}} \cdot\left(\mathbf{r}_{\mathrm{I}}-\mathbf{r}_{\mathrm{H}}\right)
$$

where: $(\vec{\nabla} \varphi)_{\mathrm{H}}$ is calculated by:

$$
(\vec{\nabla} \varphi)_{\mathrm{H}}=\frac{1}{\mathrm{~V}_{\mathrm{H}}} \quad \sum_{\mathrm{f}=\text { faces of the host cell on the donor mesh }} \varphi_{\mathrm{f}} \mathbf{A}_{\mathrm{f}}
$$

$\varphi_{\mathrm{f}}$ is approximated at the face center of the host cell using LI except in the case of pressure, where $\mathrm{P}_{\mathrm{f}}$ is approximated using PLI (Jahanbakhsh et al., 2007).

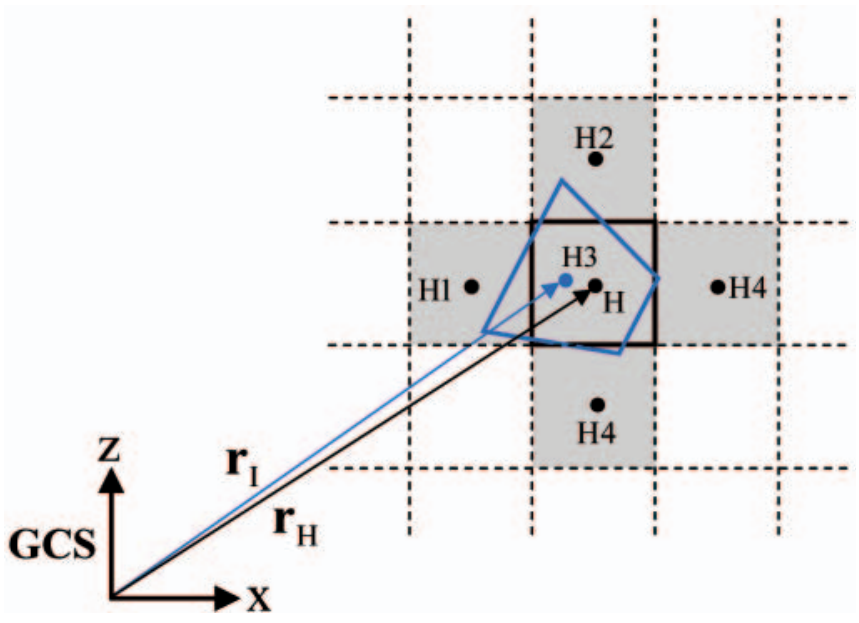

Fig. 5. An interpolation cell in the overset mesh and its interpolation stencil in the related overlap mesh consisting of a host cell and four neighbor CVS

\section{Solution of Discrete Equations}

There are two main ways to solve discrete equations on an overlapping mesh system:

次 go back and forth between mesh components (Drakakis et al., 2001)

* solve all mesh components simultaneously (Hadzic, 2005)

Using the former approach, information exchanging between meshes has a lag by an outer iteration and more iterations as well as stronger under-relaxation may be required to achieve a converged solution. In addition, to obtain a consistent pressure field in the entire domain, the reference pressure on each mesh component needs to be corrected in such a way that the pressure levels on all meshes are compatible with each other.

Having all this in mind, the later approach is implemented in this study. Here, a global matrix has to be constructed including all cells of available meshes. The procedure includes preparing the equations in all meshes and then, renumbering the overset mesh by a simple shift as the total number of cells in the background mesh to assemble a global matrix.

Assume A and B as the background and the overset meshes, respectively. Equation for a discretisation cell (D) of A mesh is:
$\mathrm{a}_{\mathrm{D}-\mathrm{A}} \varphi_{\mathrm{D}-\mathrm{A}}=\sum_{\mathrm{ngb}=\text { neighbor } \mathrm{CVs} \text { in A mesh }} \mathrm{a}_{\mathrm{ngb}-\mathrm{A}} \varphi_{\mathrm{ngb}}+\mathrm{S}_{\mathrm{D}-\mathrm{A}}$

Equation for an interpolation cell (I) of A mesh Eq.(4) is also rearranged to represent a form similar to that of a discretisation cell based on its interpolation stencil on $\mathrm{B}$ mesh:

$$
\mathrm{a}_{\mathrm{I}-\mathrm{A}} \varphi_{\mathrm{I}-\mathrm{A}}=\sum_{\text {ngb' }=\text { host cell and its neighbor CVs in B mesh }} \mathrm{a}_{\text {ngb'-B }} \varphi_{\text {ngb' }^{\prime}-\mathrm{B}}+\mathrm{S}_{\mathrm{I}-\mathrm{A}}(7)
$$

It is obvious that interpolation cells play an implicit rule in the solution procedure. Equation for an inactive cell (IA) of A mesh is prepared as well:

$$
\mathrm{a}_{\mathrm{IA}-\mathrm{A}} \varphi_{\mathrm{AI}-\mathrm{A}}=\varphi_{\mathrm{IA}-\mathrm{A}}^{*}
$$

where:

$\mathrm{a}_{\mathrm{IA}-\mathrm{A}}=1$ and $\varphi_{\mathrm{IA}-\mathrm{A}}^{\mathrm{*}}$ is the last known value of the inactive cell.

After constructing analogous equations for B mesh, it is time to assemble the global matrix for variable $\varphi$ using new continuous cell numbering.

\section{SOLUTION ALGORITHM}

The above mentioned method to solve hydrodynamics problems consists of many components. Fig. 6 clearly shows the general relation between these elements. Using the solution algorithm, one can solve a wide variety of problems but, a most common case consists of rigid bodies with up to 3-DoF motions in the context of an interfacial flow. The route to simulate such a problem is presented in Fig. 6 by bold lines.

Here, an internal loop between the solution of NavierStokes and rigid body motion equations has a vital role in the procedure. This provides a strongly coupled solution in the domain in addition to compensation of data lack for fresh cells. These are cells which were inactive in the previous time step and become interpolation/discretisation cells in the present time step. Subsequently, they have no information while they are needed in the temporal discretisation.

\section{NUMERICAL RESULTS}

Actually, the present study is based on previous researches in the field of numerical hydrodynamics. A code was developed by implementing a body-attached/moving mesh motion simulation strategy and verified in 2D and 3D problems (Panahi et al., 2006b, Jahanbakhsh et al., 2008). Now, another strategy is under investigation.

\section{Cylinder in a Steady Unsymmetrical Current}

In this section, the steady laminar flow around a circular cylinder asymmetrically placed in a channel is considered, see Fig. 7. The parabolic velocity profile corresponding to a fully developed laminar flow in a channel is prescribed at the inlet:

$$
\mathrm{u}=\frac{6 \mathrm{U}}{\mathrm{H}^{2}}\left[(\mathrm{z}+2 \mathrm{D}) \mathrm{H}-(\mathrm{z}+2 \mathrm{D})^{2}\right] ; \mathrm{w}=0
$$

where:

$\mathrm{U}, \mathrm{D}$ and $\mathrm{H}=4.1 \mathrm{D}$ are mean velocity, cylinder diameter and channel height, respectively.

The velocity gradient is equal to zero at the outlet and its magnitude is equal to zero at the cylinder surface as well as channel walls. The pressure gradient is also equal to zero 


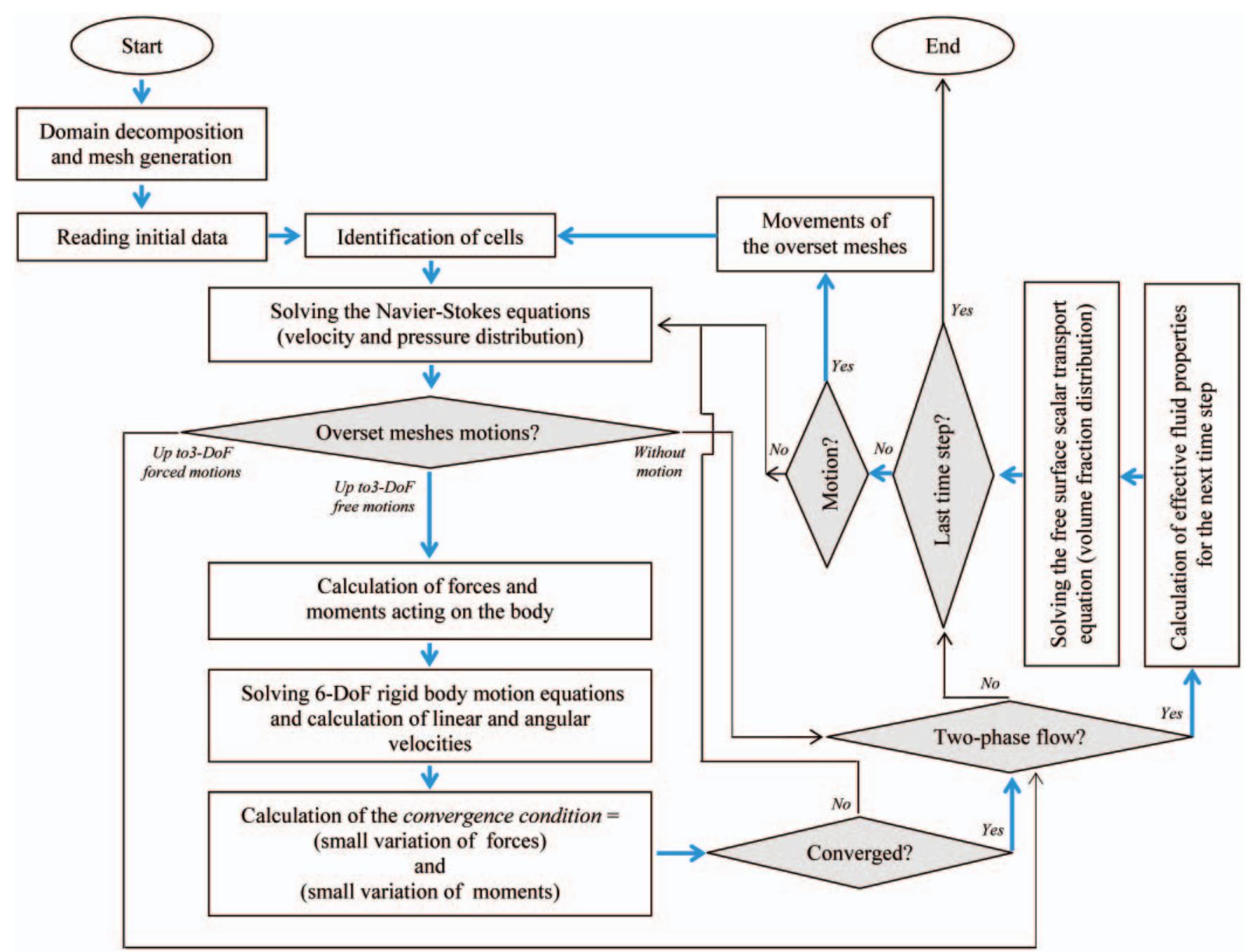

Fig. 6. The solution procedure used to develop a numerical hydrodynamics laboratory

at all boundaries. The flow domain dimensions and the fluid properties used in the computation are as follows: $\mathrm{D}=0.1 \mathrm{~m}$, $\mathrm{U}=0.2 \mathrm{~m} / \mathrm{s}, \rho=1 \mathrm{~kg} / \mathrm{m}^{3}$ and $\mu=0.001 \mathrm{~kg} / \mathrm{m}^{3}$. The Reynolds number based on the mean inlet velocity and the cylinder diameter is $\operatorname{Re}=\rho \mathrm{U} / \mu=20$. The flow is slightly asymmetric since the cylinder center is not on the horizontal symmetry plane of the channel. Due to asymmetry, different flow rates and different pressures appear above and below the cylinder, resulting in a small lift force.

For the analysis of spatial discretization errors, the computation has been performed on three systematically refined meshes, using $\delta_{0}$ of $0.062,0.034$ and $0.018 \mathrm{~m}$, respectively. The first level overset mesh has 32 uniformly distributed $\mathrm{CVs}$ around the cylinder and $10 \mathrm{CVs}$ in the radial direction. However, finer meshes are obtained by doubling the number of cells in each direction. The thickness of the cell next to the wall, in the direction normal to the cylinder surface is 0.03 , 0.00142 and 0.00069 in three levels of mesh refinement. In addition, the first level background mesh has $20 \mathrm{CVs}$ in $\mathrm{z}$ direction and $46 \mathrm{CVs}$ in $\mathrm{x}$ direction. The mesh is stretched in $z$ direction to get better resolution near the channel walls. In the $\mathrm{x}$ direction, the mesh is uniform in front of the cylinder and up to $2 \mathrm{D}$ behind the cylinder. Thereafter, the mesh is coarsened towards the outlet boundary. Cell identity using aforementioned overlapping zone width, is shown in Fig. 8. It is so important to tune $\delta_{0}$ as no interpolation cell of the overset mesh is included in the interpolation stencil constructed for an interpolation cell of the background mesh and vice versa.

Here, drag coefficient is $\mathrm{C}_{\mathrm{D}}=\mathrm{F}_{\mathrm{x}} / 1 / 2 \rho \mathrm{U}^{2} \mathrm{D}$ and lift coefficient is $\mathrm{C}_{\mathrm{L}}=\mathrm{F}_{\mathrm{z}} / 1 / 2 \rho \mathrm{U}^{2} \mathrm{D}$; where: $\mathrm{F}_{\mathrm{x}}$ and $\mathrm{F}_{\mathrm{z}}$ are the total forces on the

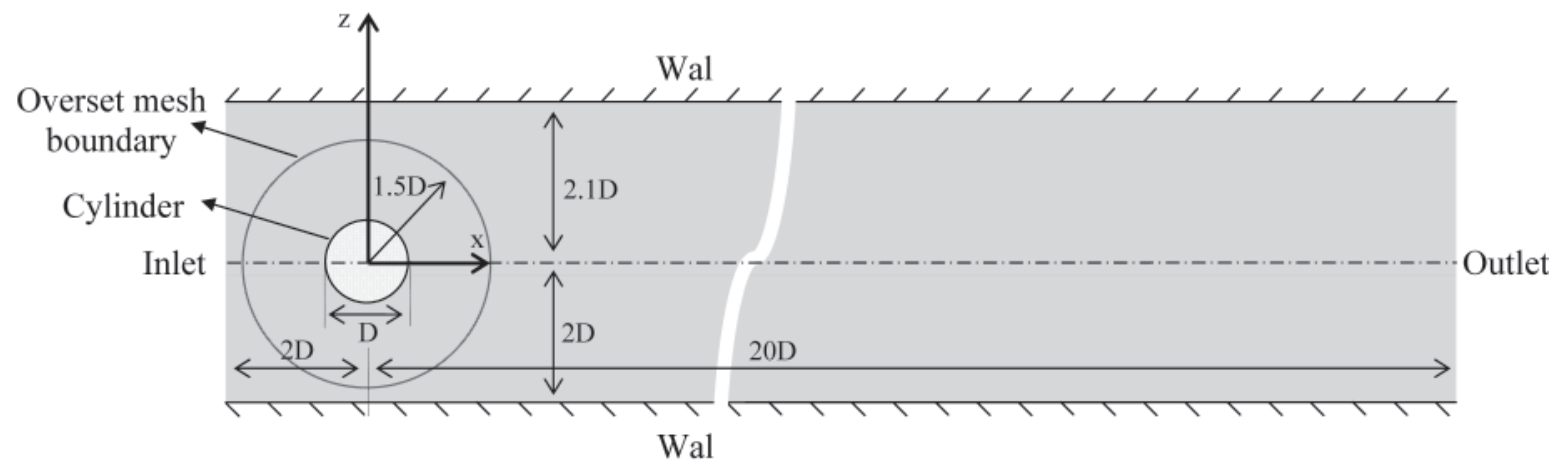

Fig. 7. Geometry and computational characteristics for laminar flow around a cylinder in a channel 


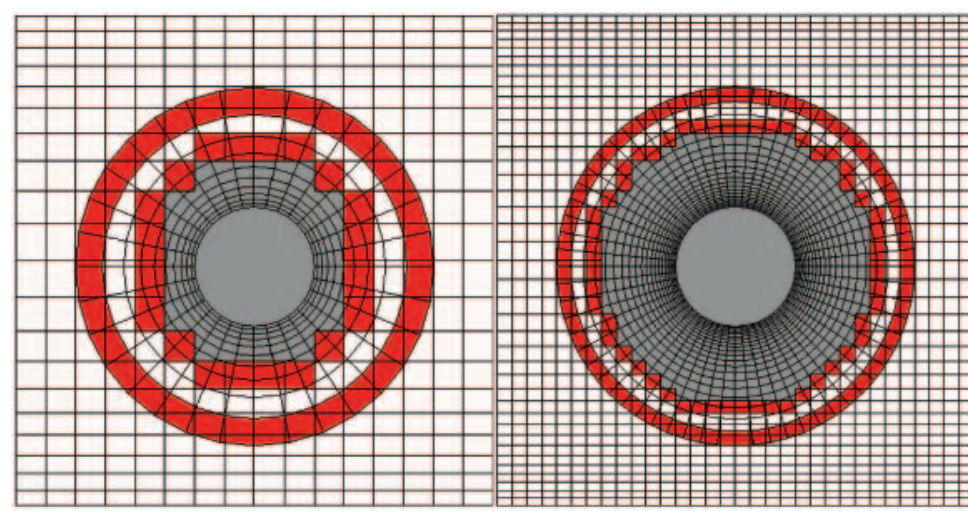

(a) (b)

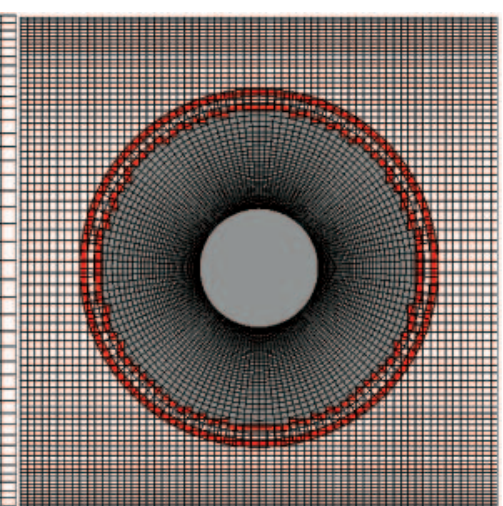

(c)

Fig. 8. Overlapping meshes used for the computation of the flow around a cylinder in a channel at first 4D of the channel length; a) rough mesh of $1240 \mathrm{CVs}, \boldsymbol{b})$ medium mesh of $4960 \mathrm{CVs}$; c) fine mesh of $19840 \mathrm{CVs}$; White CVs: discretisation cells, red CVs: interpolation cells and grey CVs: inactive cells of the background mesh

Tab. 3. Drag coefficient $C_{D}$ and lift coefficient $C_{L}$ as a function of mesh fineness

\begin{tabular}{|c|c|c|c|c|c|c|c|}
\hline \multicolumn{4}{|c|}{ Current Numerical Simulation } & \multicolumn{2}{c|}{$\begin{array}{c}\text { Benchmark } \\
\text { (Schäfer and Turek, 1996) }\end{array}$} & \multicolumn{2}{c|}{$\begin{array}{c}\text { Error in Comparison } \\
\text { to the Fine Mesh }\end{array}$} \\
\cline { 1 - 4 } Mesh & Number of CVs & $\mathrm{C}_{\mathrm{D}}$ & $\mathrm{C}_{\mathrm{L}}$ & $\mathrm{C}_{\mathrm{D}}$ & $\mathrm{C}_{\mathrm{L}}$ & $\mathrm{C}_{\mathrm{D}}$ & $\mathrm{C}_{\mathrm{L}}$ \\
\cline { 1 - 4 } rough & 1240 & 5.40077 & 0.01183 & & & & \\
\cline { 1 - 4 } medium & 4960 & 5.53412 & 0.01161 & \multirow{2}{*}{5.5800} & 0.0107 & $0.19 \%$ & $0.18 \%$ \\
\cline { 1 - 3 } fine & 19840 & 5.56939 & 0.01072 & & & & \\
\hline
\end{tabular}

cylinder in $\mathrm{x}$ and $\mathrm{z}$ directions. They are obtained to investigate the accuracy of the method and summarized in Tab. 3 in addition to a benchmark (Shäfer and Turek, 1996). All results are very close to each other and they are in a very good agreement with the benchmark data. The difference between solutions on consecutive meshes is reducing by about a factor of four, which is in accordance with expectations of a second-order discretisation. This is good news while interpolation cells are also included in the solution. In other words, the interpolation scheme has no negative effect on the total accuracy of the solution and the accuracy of spatial discretisation is maintained.

A more detailed view of the flow field at the first 13D of the channel length is given in Fig. 9 which shows the pressure and also $u$ velocity distribution in the channel in the case of the fine mesh. The overset mesh boundary is also shown in this figure. Although two different meshes are used in the overlap zone, there is almost no difference between the contours on two mesh components. Slight difference that appears near the overset mesh boundary is due to the lack of the flow

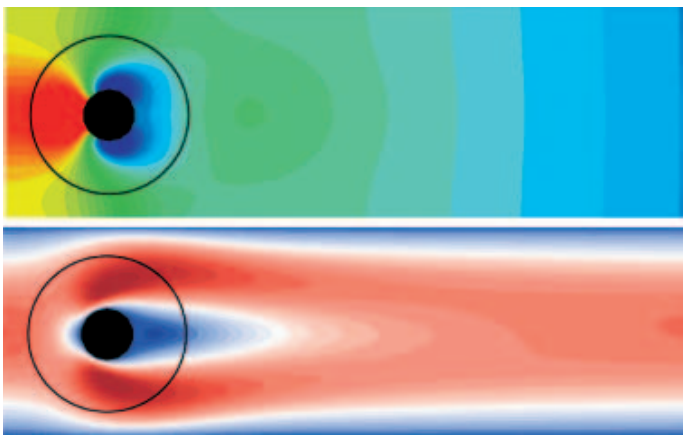

Fig. 9. Pressure field (up) and u velocity field (down) at first $13 D$ of the channel length obtained by an overlapping mesh system of $19840 \mathrm{CVS}$; circle shows boundary of the overset mesh around the cylinder; continuity of contours across the overset mesh boundary obviously shows the performance of implemented interpolation scheme for both pressure and velocity information at the boundary points during the postprocessing (presentation of results). Smooth representation of the flow field in the overlap zone confirms that the interpolation scheme introduced in this study provides a correct coupling between the mesh components and leads to a unique solution over the whole domain.

\section{Wedge-Type Wave Generator}

Here, a plunger wavemaker (Tanizawa and Clément, 2000) is simulated to validate the method in the case of a forced body motion, see Fig. 10. The wedge has a sinusoidal vertical motion of $\mathrm{z}=\sin (\sqrt{9.81} \mathrm{t})$ where the overset mesh also follows its motion. While, the background mesh remains stationary during the wave generation.

No-slip and zero-gradient boundary conditions are applied for velocity and pressure at all boundaries, respectively. Besides, in order to minimize the reflection of the flow from the right wall of the wave tank, a damping zone is considered through the last 16d of its length (Park and Miyata, 2001), see Fig. 10. Width of the overlap zone is set to $\delta_{0}=0.25 \mathrm{~m}$ and the time step is $0.002 \mathrm{~s}$. Snapshots of the free surface are illustrated after the beginning of wavemaker harmonic motion in Fig. 11.

Besides, Fig. 12 shows comparisons of the results with numerical reference data from the ISOPE Workshop (Tanizawa and Clément, 2000). The importance of temporal discretisation scheme is also shown in Fig. 13 by comparison of two generated waves. It is obvious that using the three-time-levels temporal discretisation scheme for the unsteady term of the volume fraction transport equation has a vital role to minimize the numerical diffusion in the wave tank in comparison to that of Euler implicit. Besides, another deficiency when using Euler implicit discretisation is a numerical increasing of the wave period. It must be also reported that, using the threetime-levels temporal discretisation for the unsteady term of the momentum conservation equation has not significant effects in this case. 


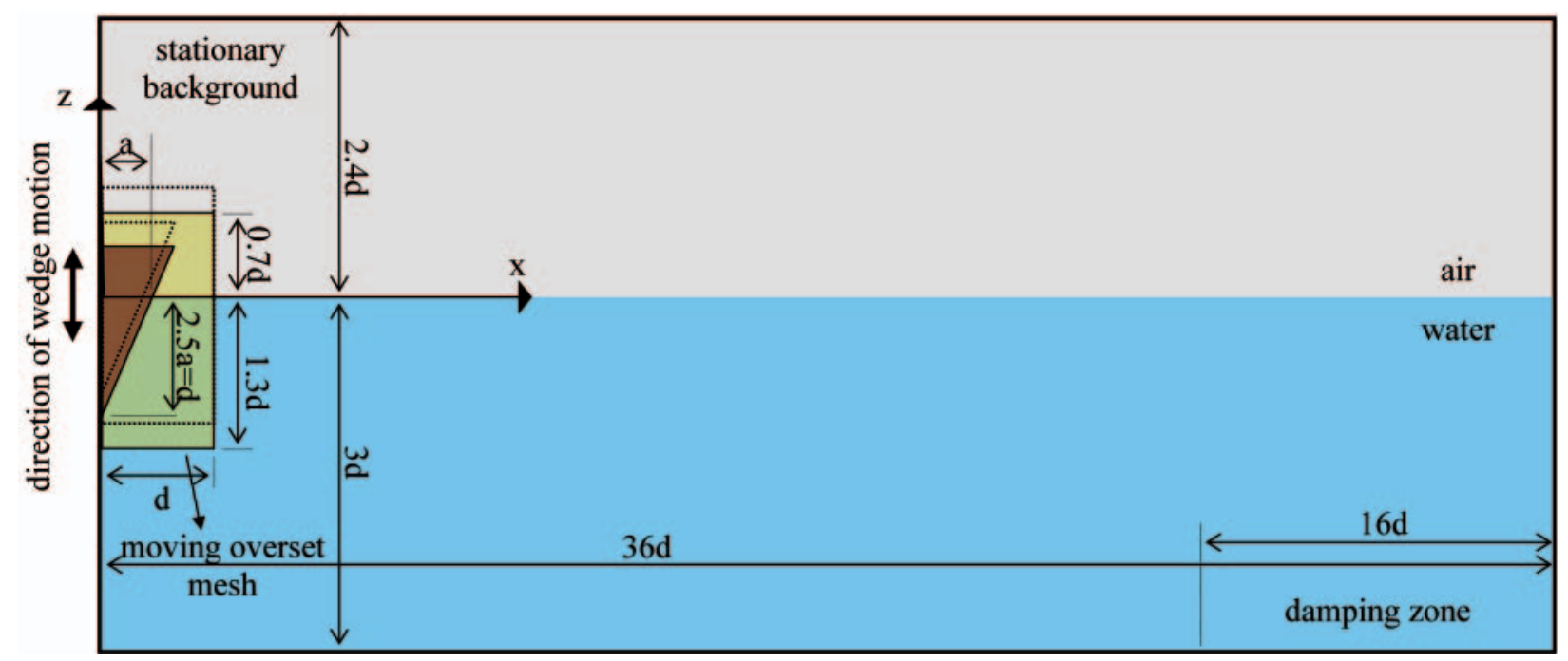

Fig. 10. Plunger wavemaker; schematic view of the computational domain including an overset mesh of $16000 \mathrm{CVS}$ with a vertical sinusoidal motion and a stationary background mesh of $75000 \mathrm{CVs} ; a=1$
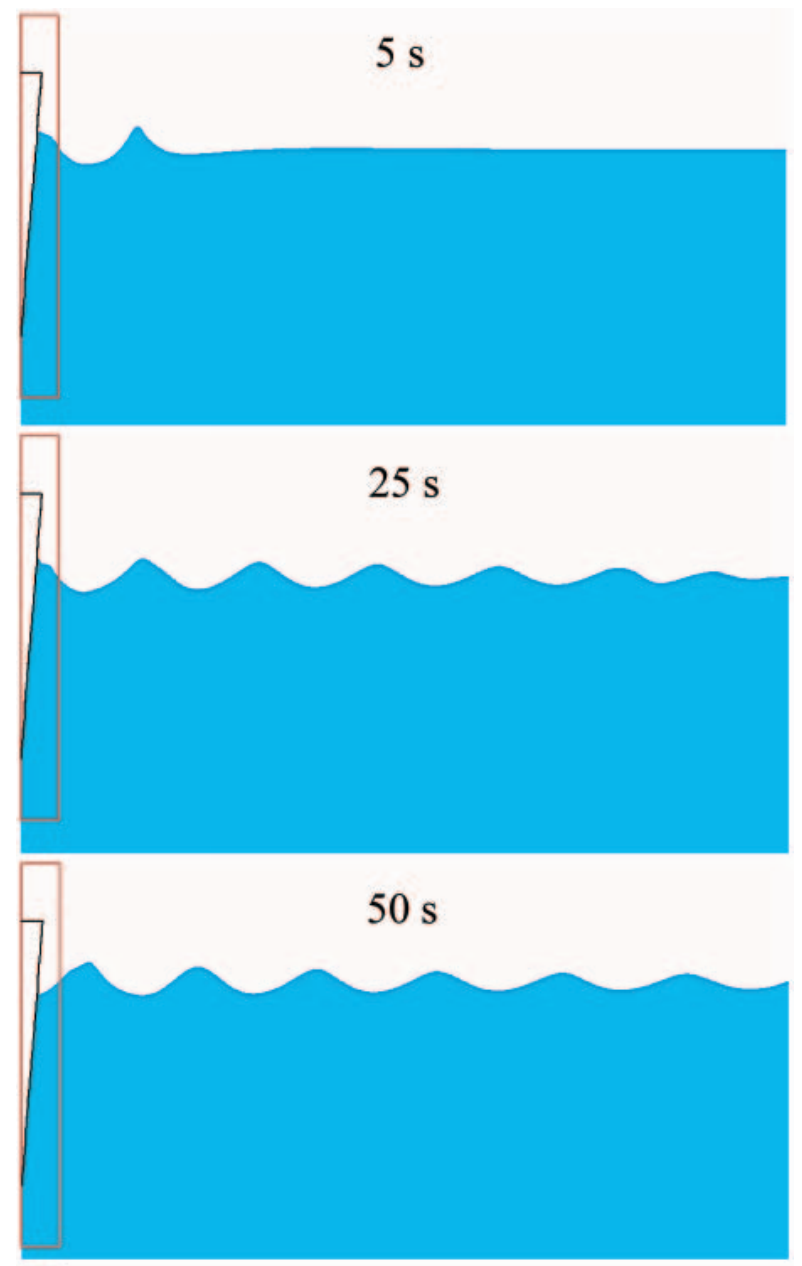

$87.5 \mathrm{~s}$

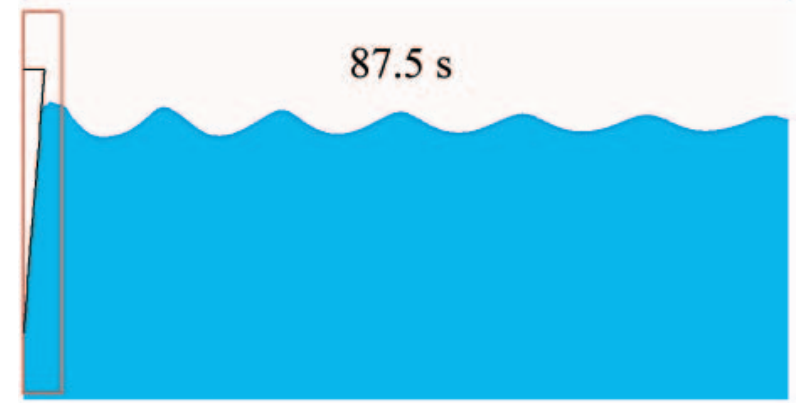

Fig. 11. Snapshots of the free surface after the beginning of plunger vertical oscillations in the first $50 \mathrm{~m}$ of the tank

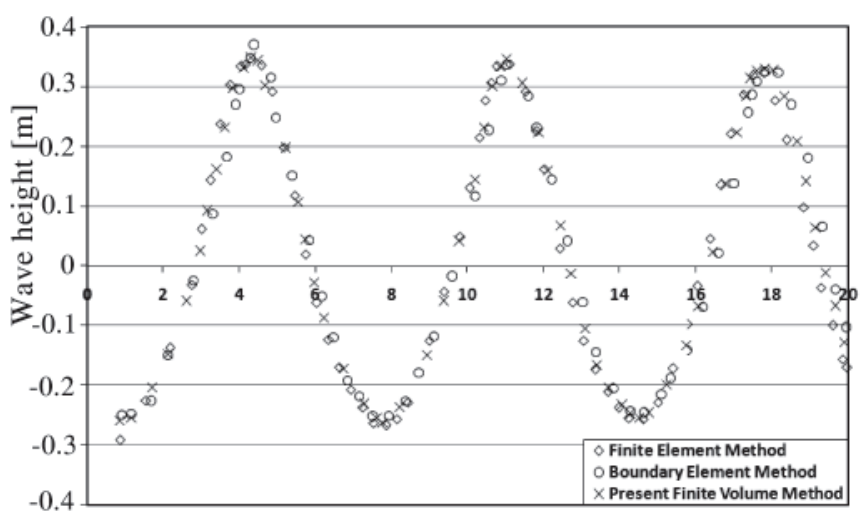

Fig. 12. Comparison of wave profiles close to the wedge with wedge at its mean position moving up; results of a Boundary Element (BEM) and Finite Element Methods (FEM) are extracted from ISOPE workshop (Tanizawa and Clément, 2000)

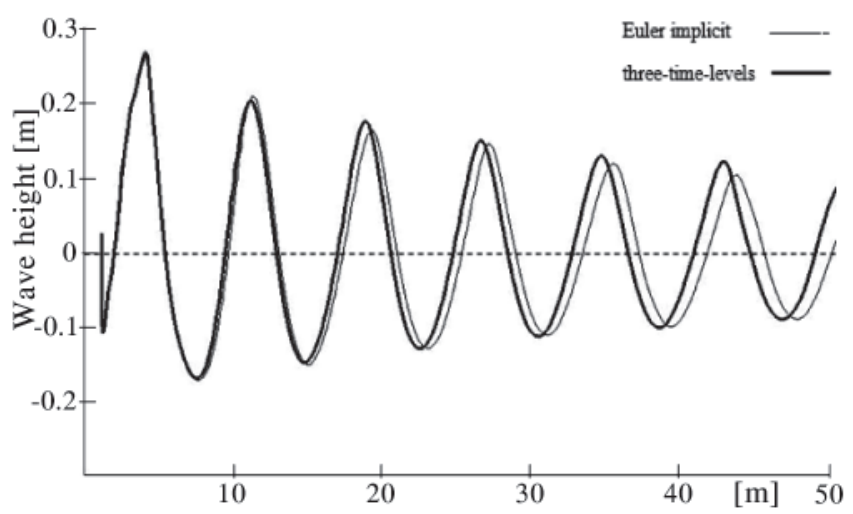

Fig. 13. The importance of implementing the three-time-levels temporal scheme to discretise the unsteady term of the volume fraction transport equation in order to capture a high quality wave tank; wave propagation at $t=80 \mathrm{~s}$

\section{Cylinder Free Falling}

To evaluate the method in the case of a free body motion, water entry of a neutrally-buoyant circular cylinder is studied, see Fig. 14. The cylinder is released from a position just above the still water level. It intersects the water surface with the downward velocity of $4 \mathrm{~m} / \mathrm{s}$. Here, no-slip boundary condition at cylinder wall, zero value at down boundary and zero-gradient at other boundaries are applied on velocity. Also, zero-gradient 
condition is used for pressure at whole boundaries. Width of the overlap zone is set to $\delta_{0}=0.02 \mathrm{~m}$ and time step is $0.0001 \mathrm{~s}$.

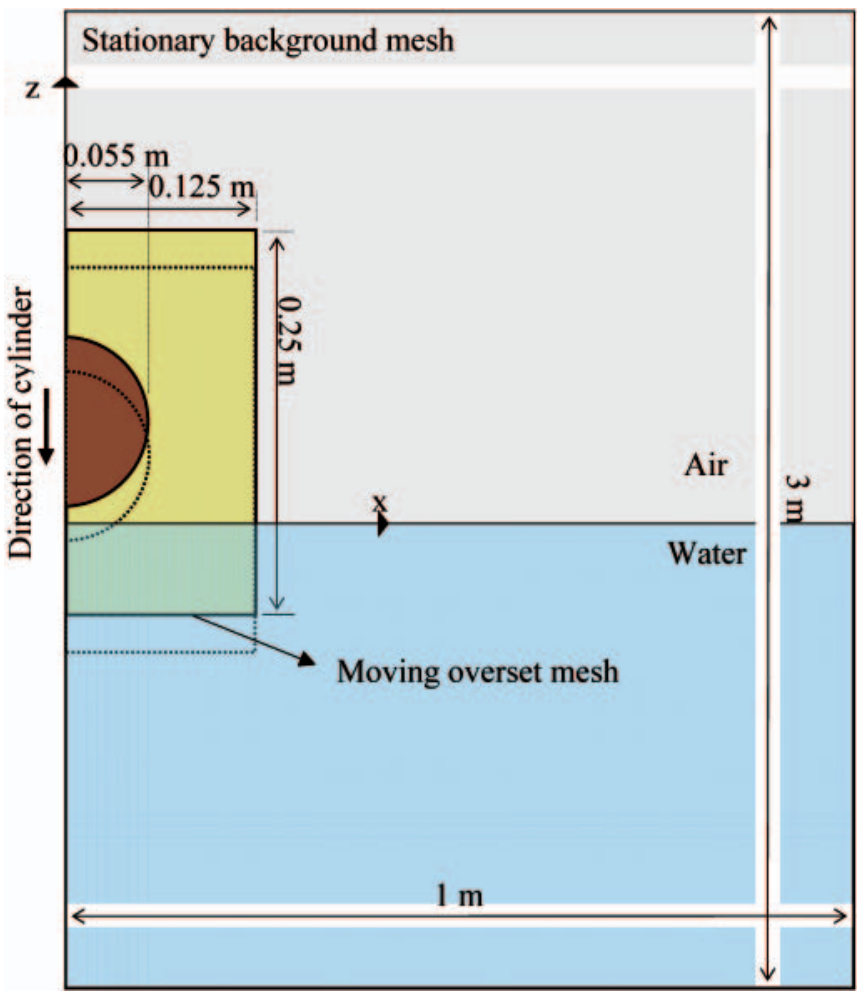

Fig. 14. Free falling cylinder; schematic view of the computational domain including an overset mesh of $6000 \mathrm{CV}$ shich follows the cylinder and a stationary background mesh of $20000 \mathrm{CVS}$

Identity of cells is shown at a time step in Fig. 15. It is obvious that the cells in the background mesh are four times larger than those in the overset mesh in the overlap zone. This announces a low sensitivity to have a similar mesh quality in the overlap zone as is a common case when using an overlapping mesh system. It is actually an important capability which facilitates the use of a high quality mesh for a body irrespective of the quality of the background mesh. Besides, it has a high value in the case of a moving body and helps to reduce the number of cells in the background mesh, while a desired resolution can be implemented in the vicinity of a moving body.

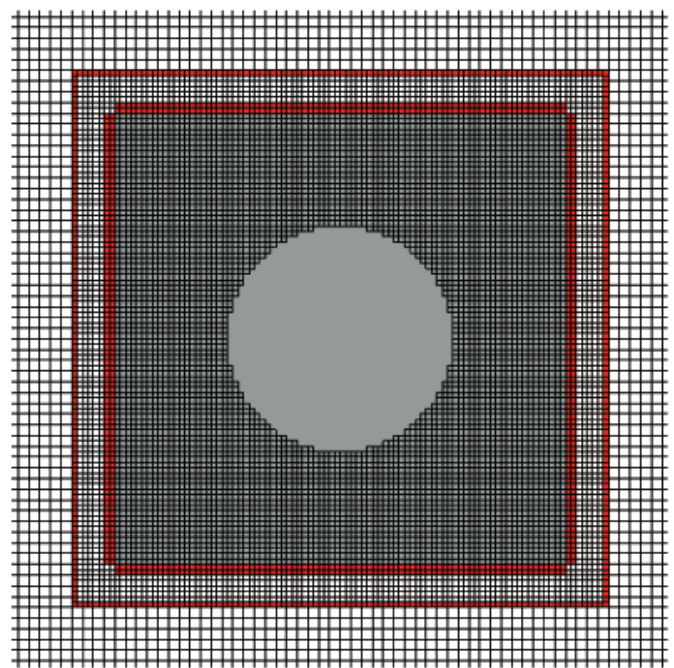

Fig. 15. Overlapping mesh used to compute the free falling problem in the vicinity of the overlap zone; cells of the background mesh are four times larger than those in the overset mesh in this region; white CVs. discretisation cells, red $C V_{s}$ : interpolation cells and grey $C V_{s}$ : inactive cells of the background mesh
After the cylinder impacts on the calm water surface, the velocity of cylinder is decreased significantly due to the effects of hydrodynamic impact forces. As shown in Fig. 16 for three time instants, water sprays are thrown up at each side of the cylinder and travel straight upward until they become unstable. As mentioned earlier, using overlapping mesh motion strategy, a problem is solved on more than one mesh. Such a solved flow field in a time step is typically shown in Fig. 17. It is obvious that the data transfer procedure is perfectly constructed. Also, the overset mesh boundary is placed in presence of large changes in the flow field. Fig. 18 also shows the time history of vertical displacement of the cylinder. The instantaneous vertical positions of the cylinder are compared with experimental data of (Greenhow and Lin, 1983) and numerical simulation of (XingKaeding, 2004; Panahi et al., 2006a). It shows a reasonably good agreement with experimental data in comparison to two numerical studies using a single body-attached mesh. It is probably due to a better quality of the mesh in the vicinity of the cylinder and also minimizing the errors due to CVs motions. Overlapping mesh system divides the domain simplifying the procedure to generate a set of high quality meshes. Consequently, it includes much less moving CVs than in the case of a moving mesh motion modeling strategy where the whole computational domain moves.
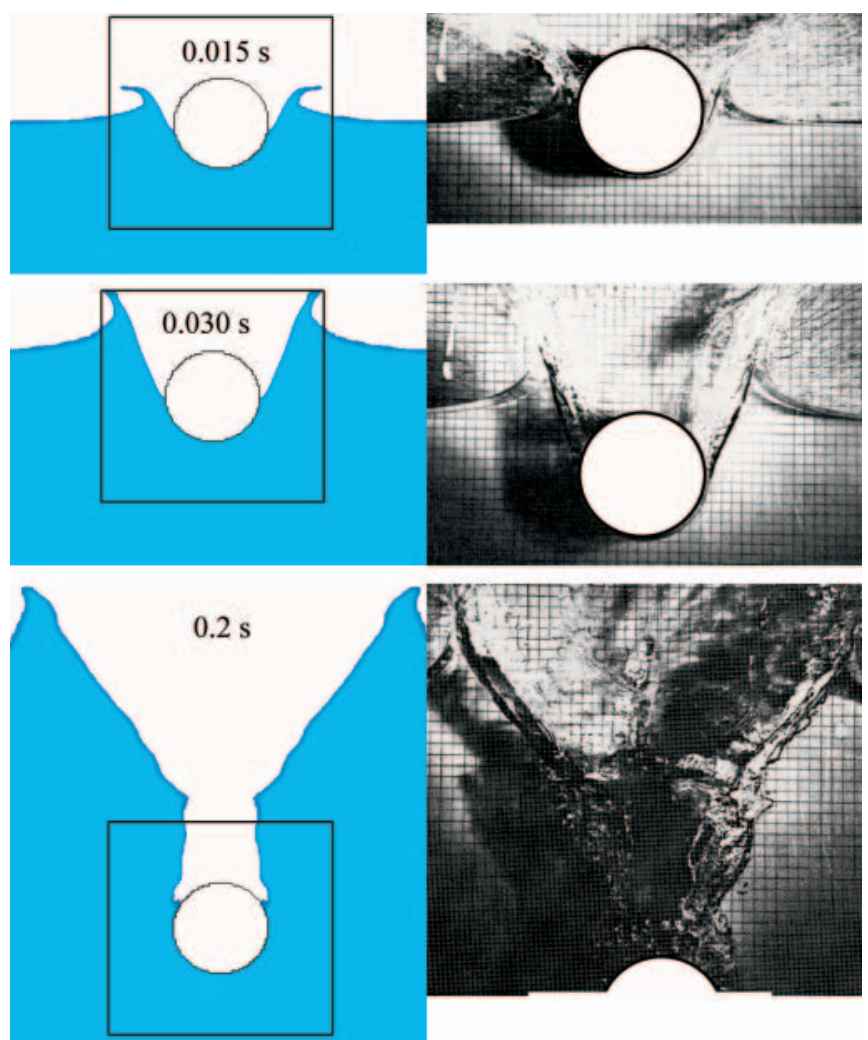

Fig. 16. Free surface deformation in cylinder water-entry problem; (left): numerical simulation using the overlapping mesh system, (right). experimental data (Greenhow and Lin, 1983)

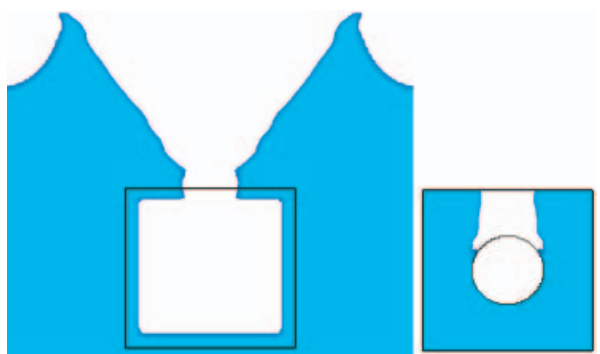

Fig. 17. Problem is solved in two mesh components using the overlapping mesh system; free surface deformation in the case of cylinder water-entry is presented at $t=0.2 \mathrm{~s}$ in the stationary background mesh (left) and the moving overset mesh (right) 


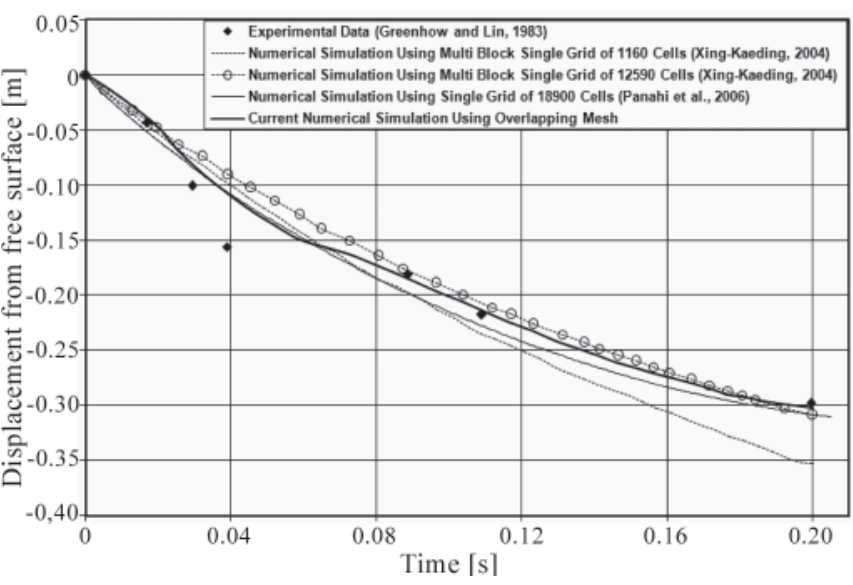

Fig. 18. Time history of the cylinder water-entry just after the impact

\section{CONCLUSION}

As a complementary tool to marine laboratory tests, CFD proposes the ability to study a wide variety of hydrodynamic problems using an integrated method. Here, an algorithm among such possibilities is developed based on FV overlapping mesh system to deal with two-phase flow interaction with a structure. Selected test cases are good problems to assess different aspects of the proposed method. It can be simply developed to solve more complete problems especially to record hydrodynamics behavior of more than one structure in a numerical wave tank. Besides, the algorithm can be easily extended to 3D problems.

\section{BIBLIOGRAPHY}

1. Atta E.H.: Component adaptive grid interfacing. AIAA Paper, 81-0382, 1981

2. Blades E., Marcum D.L.: A sliding interface method for unsteady unstructured flow simulations. International Journal for Numerical Methods in Fluids, 2007

3. Buning P.G., Wong T.C., Dilley A.D., Pao J.L.: Prediction of hyper-x stage separation aerodynamics using CFD. AIAA Paper, 2000-4009, 2000

4. Carrica P.M., Wilson R.V., Noack R.W., Stern F.: Ship motions using single-phase level set with dynamic overset grids. Computers \& Fluids, 36(9), 2007

5. Chen H.C., Lin W.M., Hwang W.Y.: Validation and application of chimera RANS method for ship-ship interactions in shallow water and restricted waterway. $24^{\text {th }}$ ONR Symposium on Naval Hydrodynamics, Fukuoka, Japan, 2002

6. Chentanez N., Goktekin T.G., Feldman B.E., O'Brien J.F.: Simultaneous coupling of fluids and deformable bodies. Proceedings of the 2006 ACM SIGGRAPH/Eurographics Symposium on Computer Animation, 2006

7. Drakakis D., Majewski J., Rokichki J., Zoltak J.: Investigation of blending-function-based overlapping-grid technique for compressible flows. Computer Methods in Applied Mechanics and Engineering. 190, 2001

8. Ferziger J., Peric M.: Computational methods for fluid dynamics. $3^{\text {rd }}$ Rev. Ed., Springer Verlag, 2002

9. Greenhow M., Lin W.: Nonlinear free surface effects: experiments and theory. Report No. 83-19, Massachusette Institute of Technology, 1983

10.Hadzic H.: Development and application of a finite volume method for the computation of flows around moving bodies on unstructured, overlapping grids. $\mathrm{PhD}$ Thesis, Teschnichen Universitat Hamburg-Harburg, 2005

11.Jahanbakhsh E., Panahi R., Seif M.S.: Numerical simulation of three-dimensional interfacial flows. International Journal of Numerical Methods for Heat \& Fluid Flow, 17(4), 2007
12. Jahanbakhsh E., Panahi R., Seif M.S.: Catamaran motion simulation based On moving grid technique. Journal of Marine Science and Technology, Accepted for Publication, June 2008

13.Jasak H.: Error analysis and estimation for finite volume method with application to fluid flows. $\mathrm{PhD}$ Thesis, University of London, 1996

14.Kim D., Choi H.: A second-order time-accurate finite volume method for unsteady incompressible flow on hybrid unstructured grids. Journal of Computational Physics, 162(2), 2000

15.Löhner R.: Robust, vectorized search algorithms for interpolation on unstructured grids. Journal of Computational Physics, 118(2), 1995

16.Meakin R.L.: Object X-rays cutting holes in composite overset structured grids. AIAA Paper, 2001-2537, 2001

17.Mittal M., Iaccarino G.: Immersed boundary methods. Annual Review of Fluid Mechanics, 37, 2005

18.Nakahashi K., Togashi F., Sharov, D.: An intergrid-boundary definition method for overset unstructured grid approach. AIAA Journal, 38(11), 2000

19.Panahi R., Jahanbakhsh E., Seif M.S.: Comparison of interface capturing methods in two phase flow. Iranian Journal of Science \& Technology, Transaction B: Technology, Vol.29, No.B6, 2005

20.Panahi R., Jahanbakhsh E., Seif M.S.: Development of a VoF fractional step solver for floating body motion simulation. Applied Ocean Research, 28(3), 2006a

21.Panahi R., Jahanbakhsh E., Seif M.S.: Numerical investigation on the effect of baffle arrangement in tanker sloshing. ${ }^{\text {th }}$ Numerical Towing Tank Symposium (NuTTs), Nantes, France, $2006 \mathrm{~b}$

22.Park J.C., Miyata H.: Numerical simulation of fully-nonlinear wave motions around arctic and offshore structures. Journal of the Society of Naval Architects of Japan, 189, 2001

23.Schäfer M., Turek S.: Benchmark computations of laminar flow around a cylinder $[J]$. Notes on Numerical Fluid Mechanics, 52, 1996

24.Suhs N.E., Rogers S.E., Dietz W.E.: PEGASUS: An Automated pre-processor for overset grid CFD. AIAA Paper, 2002-3186, 2002

25.Tanizawa K., Clément A.H.: Report of the $2^{\text {nd }}$ workshop of ISOPE numerical wave tank group: benchmark test cases of radiation problem. $10^{\text {th }}$ International Offshore and Polar Engineering Conference, Seattle, USA, 2000

26.Togashi F., Nakahashi K., Ito Y., Iwamiyata T., Shimbo Y.: Flow simulation of NAL experimental supersonic airplane/booster separation using overset unstructured grids. Computers and Fluids, 30(6), 2001

27.Tremel U., Sorensen K.A., Hitzel S., Rieger H., Hassan O., Weatherill N.P.: Parallel remeshing of unstructured volume grids for CFD applications. International Journal for Numerical Methods in Fluids, 53(8), 2007

28.Ubbink O., Issa R.I.: A method for capturing sharp fluid interfaces on arbitrary meshes. Journal of Computational Physics, 153(1), 1999

29.Xing-Kaeding Y.: Unified approach to ship seakeeping and maneuvering by a RANSE method. PhD Thesis, Teschnichen Universitat Hamburg-Harburg, 2004.

\section{CONTACT WITH THE AUTHORS}

Roozbeh Panahi, Assoc. Prof. Mehdi Shafieefar, Assoc. Prof.

Civil Engineering Department, Tarbiat Modares University,

P.O. Box: 14155-4838, Tehran, Iran, tel: +982182883318 , fax: +9821 82883538, email: shafiee@modares.ac.ir 